\title{
A Transmission Line Method for the Simulation of Fiber Bragg Gratings
}

\author{
N. A. Stathopoulos ${ }^{1 *}$, S. P. Savaidis ${ }^{1}$, H. Simos ${ }^{1}$, E. Rigas ${ }^{2}$, R. Correia ${ }^{2}$, \\ S. W. JAMES ${ }^{2}$, R. P. TATAM ${ }^{2}$ \\ ${ }^{1}$ University of West Attica, Department of Electrical and Electronics Engineering, 12244 Aegaleo, Athens, Greece \\ ${ }^{2}$ Centre of Engineering Photonics - Cranfield University UK \\ *Corresponding author: nstath@uniwa.gr
}

Received XX Month XXXX; revised XX Month, XXXX; accepted XX Month XXXX; posted XX Month XXXX (Doc. ID XXXXX); published XX Month XXXX

\begin{abstract}
A new method for the analysis and design of fiber Bragg gratings (FBG) based on the theory of transmission lines has been developed and verified both theoretically and experimentally. The method is an extension of the Coupled Mode Theory and utilizes the equivalent transmission lines in order to simulate any type of grating, with an easy and direct implementation. The method provides the ability to analyze the optical devices without using full wave approaches, while also facilitating the incorporation of core materials with a complex or nonlinear refractive index, non-uniform distributions of the grating's refractive index, and tilted and phase shifted gratings. The approach also allows the design of the grating for a given reflection spectra. Numerical results of the method's application on a randomly varied inscription of the refractive index of a FBG have also been simulated and discussed. Using this method, the characteristics of an Erbium - Doped FBG have been simulated and the predictions verified experimentally.
\end{abstract}

OCIS codes: (060.3735) Fiber Bragg gratings; (060.3738) Fiber Bragg gratings, photosensitivity (000.4430) Numerical approximation and analysis; (080.2720) Mathematical methods (general).

http://dx.doi.org/10.1364/A0.99.099999

\section{Introduction}

Fibre Bragg Gratings (FBG) have been particularly popular in the field of optical communication systems, mainly as optical reflectors, filters, multiplexers / demultiplexers and dispersion compensation devices [1-5]. More important however, is their use as sensors as they exhibit many and important advantages over traditional electrical sensors, offering high precision, stability over time, immunity to electromagnetic interference, support for long range detection, suitability for use in explosive environments, and inherently offering multiplexed sensing via a single fiber-optic channel [6-8]. Because of their advantages they are applied in a wide variety of fields such as industrial automation, energy, automotive, aerospace, security and even medicine [9-10]. The use of FBG allows measurements of physical quantities and parameters such as mechanical stresses, displacement, pressure and temperature change [4].

The numerical analysis and synthesis of an FBG is mainly concentrated on the optical intensity spectral reflectivity and the dominant method of analyzing these devices is the Coupled Mode Theory (CMT) [11-13]. This method is accurate and consistent for all uniform gratings and with appropriate modifications and, in combination with the Transfer Matrix Method (TMM), may be used to analyze uniform and nonuniform gratings with a complex refractive index i.e. with gain or loss [14]. A typical limitation of the method, which may increase its complexity and reduce its accuracy, is its application on non-uniform distributions in both the amplitude and the periodicity of the refractive index inscription in the core, as the segmentation of the structure in tandem uniform segments is required. An additional characteristic of the method is the pure mathematical formation of the analysis, which cannot always provide a physical insight to the problem.

Within the framework of this paper we propose the use of a Transmission Line Method (TLM) for the numerical analysis of FBGs. The herein proposed TLM is an extension of the CMT method since it is based on the original theory of coupling between the guided modes [15]. By analyzing the coupling between the propagation modes with and without the variation of the refractive index of the core, differential equations are derived that correspond to differential equations of transmission lines with virtual voltage and current quantities, representing the electric and magnetic fields in the grating, respectively. As a result, it is possible to simulate the whole length of the grating as a chain of transmission lines connected in tandem, where each transmission line has different characteristic impedance, depending on the refractive index in the respective point of the grating. It should be noted that in the above model there is no limitation on the form of the refractive index distribution, so it is possible to analyze an arbitrary distribution of the index in the core without modifying the model. Characteristics of the grating, such as its spectral reflectivity and transmission, as well as the distribution of the field inside the grating, are calculated in a particularly simple way, and it is easy to add losses or amplifications, nonlinearity and tilted or chirped characteristics.

The proposed TLM is characterized as a one-dimensional (1D) model, so it retains the advantages of fast methods of 
analysis while at the same time addressing accurately issues of inhomogeneity or nonlinearity on the propagation axis. By applying the transmission line method, the non-uniformity of the grating inscription along its length, due to possible imperfections such as the distribution of germanium in the fiber core or the alignment in the inscription technique, can be analyzed. This analysis may estimate the final tolerances expected during the inscription of the grating while a corresponding analysis can be made after introducing a nonlinearity of the refractive index, which can also be simulated by the proposed method.

The paper is organized as follows: Section II presents the theoretical framework for single mode fibers, which enables the FBG simulation with the use of transmission line theory. In the same section, the implementation of the TLM calculation scheme is provided for lossless gratings and its application for nonlinear, lossy or gained, chirped, and tilted grating is also described. This discussion is further extended to include the use of TLM for the design of a grating. In section III the method's accuracy is verified in comparison to the CMT method, while in the same section numerical results are provided for random variations in inscription, as well as for erbium doped gratings. In section IV an experimental verification of the method is conducted for the operation of an Erbium-doped fiber grating, while in the final section conclusions on the TLM are provided.

\section{Modeling of an FBG with a Transmission Line Method}

In this section we deploy the transmission line method in order to analyze the reflection and transmission of homogeneous or inhomogeneous FBGs with losses or gain, e.g. when an Erbium-Doped core material is used. The method may be applied for an arbitrary complex refractive index distribution along the grating. The proposed method may substitute other typical methods, such as the CMT, in order to model an arbitrary complex refractive index perturbation inside a grating, while at the same time retaining its simple, accurate and fast calculation features. Section A analyzes the theory that establishes the fundamentals for the application of the equivalent transmission lines. In Section B we describe the application of the method for the calculation of the spectral reflection for a typical uniform FBG with losses or gain.

\section{A. Equivalent Transmission Lines in Fiber Bragg Gratings}

We consider an FBG which, for the length of the grating along the $\mathrm{z}$ axis , is a perturbation (homogeneous or inhomogeneous) of the core refractive index of the single mode fiber (SMF). For multimode fibers (MMF), a similar though more complicated procedure may be followed since the coupling coefficients between the modes have to be determined. Adopting the electromagnetic description approach for the propagation of light along the $z$-axis of the fiber's core, we assume that the tangential electric and magnetic field components, $E_{t}$ and $H_{t}$, in the grating are of the following form [15]:

$$
\begin{aligned}
& E_{t}(x, y, z)=a_{1}(z) E_{1 t}(x, y) \\
& H_{t}(x, y, z)=b_{1}(z) H_{1 t}(x, y)
\end{aligned}
$$

with $E_{1 t}$ and $H_{1 t}$ being the tangential field components inside the unperturbed fiber, where the index 1 denotes that we have only one propagation mode. Additionally, $a_{1}$ and $b_{1}$ are the amplitude coefficients that modify the field components with respect to the unperturbed fiber, which are interconnected through the following equations [15]:

$$
\begin{aligned}
& \frac{d b_{1}}{d z}=\left(2 K_{11}-j \beta_{1}\right) a_{1} \\
& \frac{d a_{1}}{d z}=\left(2 k_{11}-j \beta_{1}\right) b_{1}
\end{aligned}
$$

where $K_{11}, k_{11}$ are coupling factors (calculated in the Appendix), $\beta_{1}=\bar{n} k_{0}$ is the propagation constant for the unperturbed SMF with $\bar{n}$ the effective index and $k_{0}$ the wavenumber. By integrating (1) over the $x-y$ fiber core's cross section and substituting it into (2), the following equations can be derived:

$$
\begin{gathered}
\frac{d}{d z} \iint H_{t}(x, y, z) d x d y= \\
=\left(2 K_{11}-j \beta_{1}\right) \frac{\iint H_{1 t}(x, y) d x d y}{\iint E_{1 t}(x, y) d x d y} \iint E_{t}(x, y, z) d x d y \\
\frac{d}{d z} \iint E_{t}(x, y, z) d x d y= \\
=\left(2 k_{11}-j \beta_{1}\right) \frac{\iint E_{1 t}(x, y) d x d y}{\iint H_{1 t}(x, y) d x d y} \iint H_{t}(x, y, z) d x d y
\end{gathered}
$$

Next, the auxiliary quantities of magnetic voltage and current along the propagation $z$ axis are defined as follows:

$$
\begin{aligned}
& V_{M}(z)=\iint H_{t}(x, y, z) d x d y \\
& I_{M}(z)=\iint E_{t}(x, y, z) d x d y
\end{aligned}
$$

As a result, the transmission line magnetic impedance for the corresponding unperturbed SMF is defined as follows:

$$
Z_{0 M}=\frac{\iint H_{1 t}(x, y) d x d y}{\iint E_{1 t}(x, y) d x d y}
$$

By introducing (4) and (5) into (3) we arrive at the following equations:

$$
\frac{d}{d z} V_{M}(z)=\left(2 K_{11}-j \beta_{1}\right) Z_{0 M} I_{M}(z)
$$




$$
\frac{d}{d z} I_{M}(z)=\left(2 k_{11}-j \beta_{1}\right) \frac{1}{Z_{0 M}} V_{M}(z)
$$

Equations (6) represent a magnetic transmission line along the $Z$ direction, with characteristic impedance $Z_{M}$ and transmission constant $\gamma_{M}$ as follows:

$$
\begin{gathered}
Z_{M}=Z_{0 M} \sqrt{\frac{2 K_{11}-j \beta_{1}}{2 k_{11}-j \beta_{1}}} \\
\gamma_{M}=\sqrt{\left(2 K_{11}-j \beta_{1}\right)\left(2 k_{11}-j \beta_{1}\right)}
\end{gathered}
$$

As a result, the fiber grating may be modeled as a chain of $N$ transmission lines connected in tandem with length $\Delta L$, characteristic impedance $\mathrm{Z}_{M i}$ and transmission constant $\gamma_{M i}$, respectively.

\section{B. Application of the TLM for the simulation of an FBG}

We assume a uniform sinusoidal FBG of length $L$ with variable refractive index along the grating in the core $n(z)$ according to the following form:

$$
n^{2}=\Delta n^{2} \cos \left(\frac{2 \pi}{\Lambda} z+\phi(z)\right)+n_{0}{ }^{2}
$$

where $n_{0}$ is the refractive index in the core of the fiber, $\Delta n$ represents the amplitude of the refractive index variation in the grating and $\Lambda$ is the grating period. Next, in order to apply the TLM, we consider that the length $L$ of the grating consists of $N$ thin layers each of length $\Delta L$. As will be discussed in Section II, $\Delta L$ is a fraction of $\Lambda$ but the actual value depends on the convergence of numerical results with respect to the targeted numerical accuracy. Their equivalent model is depicted in Figure 1 with $N$ transmission lines connected in tandem. The characteristic impedance $Z_{M i}$ and transmission constant $\gamma_{M i}$ for the $i$ th layer depends on $n(z)$, since the factors $K_{11}, k_{11}$ also depend on the variation of the refractive index along the $z$-axis according to (A.6-A.12).

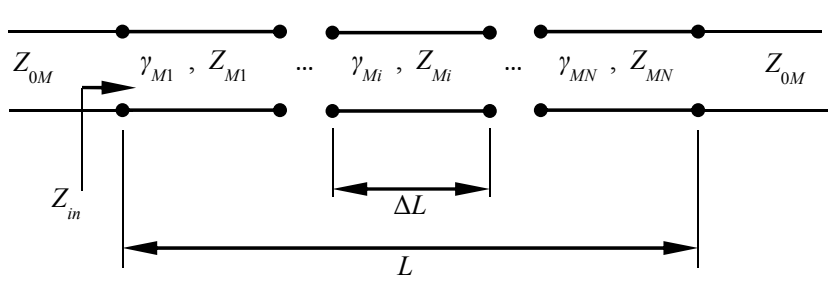

Fig. 1. Transmission lines connected in tandem for the modeling of an FBG of length $L$.

The reflection coefficient $\rho$ at the input of the transmission line circuit of Fig. 1 represents the incident light intensity reflection coefficient and can be calculated as follows:

$$
|r|=|\rho|^{2}=\left|\frac{Z_{\text {in }}-Z_{0 M}}{Z_{\text {in }}+Z_{0 M}}\right|^{2}
$$

For the calculation of $Z_{i n}$, a recursive procedure should be followed. As depicted in Fig. 2, the ith transmission line of Fig. 1 may be approximated by its ' $\mathrm{T}$ ' impedance equivalent circuit, where the series and parallel impedances are given by the following expressions:

$$
\begin{gathered}
Z_{s i}=Z_{M i} \tanh \left(\gamma_{M i} \Delta L / 2\right) \\
Z_{p i}=Z_{M i} / \sinh \left(\gamma_{M i} \Delta L\right)
\end{gathered}
$$

In the case where the refractive index of the core is real (without losses or gain), the reflection coefficient from the $i$ th transmission line is given as follows:

$$
\rho_{i-1}=\frac{Z_{i n, i}-Z_{M, i-1}}{Z_{i n, i}+Z_{M, i-1}}
$$

where $Z_{i n, i}=Z_{s i}+Z_{p i} / /\left(Z_{s i}+Z_{i n, i+1}\right)$ and may iteratively come to the calculation of $Z_{\text {in }}$ and $\rho$ in (9).

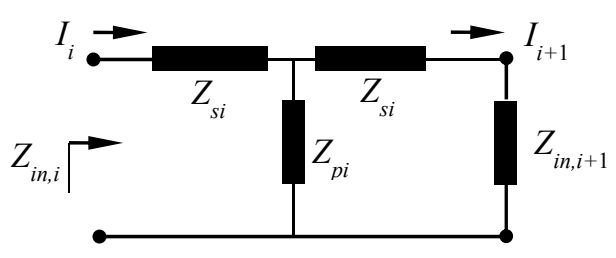

Fig. 2. Equivalent circuit of the ith transmission line of Fig.1.

If we take into account the losses or gain of the fiber core material, then the imaginary part of the refractive index may be negative (losses) or positive (gain). Assuming the imaginary part of the refractive index as $\pm j n_{i s}$ the input impedance of the ith transmission line will be modified as follows:

$$
Z_{i n, i}=Z_{M, i-1} \frac{1+\rho_{i-1}\left(1 \pm 2 n_{i s} k_{0} \Delta L\right)}{1-\rho_{i-1}\left(1 \pm 2 n_{i s} k_{0} \Delta L\right)}
$$

where, $k_{0}$ is the wavenumber for the wavelength of operation and $\rho_{i-1}$ is given by (11).

For the calculation of the optical power transmission coefficient of the grating, the current through the ' $\mathrm{T}$ ' circuit chain should be calculated, considering the end current as unity. Taking into account the boundary condition for current and voltage in the front interface of the grating the transmission coefficient is calculated as follows:

$$
|t|=\left|\frac{1-\rho^{2}}{I_{\text {in }}{ }^{2} Z_{\text {in }}}\right|
$$

where, $Z_{\text {in }}$ is calculated by (12), $\rho$ is calculated through (9) and $I_{\text {in }}$ is calculated recursively using a current division calculation scheme along the ' $\mathrm{T}$ ' circuit chain.

Furthermore, the circuit model of Fig. 2 may be used for the calculation of the field distribution across the grating using a 
recursive method for the calculation of the magnetic voltage and current along the transmission line. A profound application of the TLM capability to estimate the field distribution across the layers is the simulation of a nonlinear grating. Particularly, the refractive index within a layer may be power dependent. Consequently, the circuit elements of Fig. 2 may be calculated from the magnetic current or voltage across the layer. Following a similar procedure described in [16] for planar structure reflectors, according to which the current distribution determines the refractive index of each thin layer, the powerdepended reflectivity of the fiber grating may be simulated.

Another class of grating is the non-uniform FBG, which includes chirped FBGs (CFBG), phase shifted FBGs and tilted FBGs. For CFBGs, which are utilized for the fabrication of asymmetric optical filters, the analysis with TLM is based on (8) considering $\Lambda(\mathrm{z})$, whereas analysis with the CMT-TMM requires uniform segmentation. Phase shifted FBGs may also be analyzed using the TLM, as will be presented in the next section, while for tilted gratings and for SMF, the closed form of the refractive index variation in fiber axis, as it is described in [11], is directly applicable for TLM analysis.

The proposed model may also be applied to the synthesis of non-uniform gratings using the discrete layer-peeling (DLP) technique [17-19]. The implementation of the DLP technique for the synthesis of non-uniform gratings, which are characterized by a specified spectral reflectivity, requires its inverse Fourier transformation and its application on each of the layers that have been defined for the TLM. Thus, the refractive index of each layer may be calculated through the circuit of Fig.2, assuming that it is terminated in $\mathrm{Z}_{0 \mathrm{M}}$, and using (9), given that the reflection coefficient $\rho$ for each layer may be calculated using the algorithm described in [4].

Overall, the TLM may offer an alternative to the CMT -TMM method of analysis and synthesis of any kind of FBGs, including non-uniform and nonlinear, by avoiding adaptations that may require mathematical manipulations with respect to the basic couple-mode theory.

\section{Numerical Results}

We consider a uniform FBG of length $4 \mathrm{~mm}$ with Bragg wavelength $1530.23 \mathrm{~nm}$ and $\Delta n$ in the order of $0.04 \%$ of the core's refractive index $n_{0}=1.56$. By applying the CMT method we may calculate the spectral reflectivity that is illustrated in Fig. 3 (solid red line) for an optical fiber with effective refractive index $=1.55$. Using the TLM we may analyze this FBG by slicing its length in layers of thickness $\Delta L=\Lambda / 5$ and $\Lambda / 10$ in order to demonstrate the convergence properties of the method. The spectral power reflectivity is depicted in Fig. 3 (dashed and dotted lines respectively). It can be seen that for $\Delta L=\Lambda / 10$ an almost perfect coincidence with the CMT is obtained (dotted line). It is evident that layer thickness is a critical parameter for the application of the TLM and increases the complexity due to the increased number of layers for a specific grating length. For the examined case, the layer's thickness should be less than $\Lambda / 10$ in order to esnure the numerical accuracy of the method and, as a result, the computational complexity increases in comparison with the CMT for a uniform lossless grating. It is worth noting that the $\Delta L$ values depend on the FBG bandwidth and eventually $\Delta L$ will decrease as long as the bandwidth decreases. Nevertheless, for non-uniform, lossy or nonlinear FBG, the complexity of both CMT-TMM and TLM are of the same order, while the TLM approach is advantageous over the CMT-TMM due to the direct application of the method.

A more complex application of the proposed method concerns the analysis of an erbium-doped FBG. This device is characterized by the core's complex refractive index, where the real part follows the uniform sinusoidal form, while the imaginary part is power-depended. Particularly, the imaginary part of the refractive index may be either negative or positive and its value depends on the pump power provided by an external laser diode (LD) source.

The spectral reflectivity of the aforementioned device has been analyzed using the CMT [20] for a grating of length $4 \mathrm{~mm}$ with $\Delta \mathrm{n}$ in the order of $0.0043 \%$ of the core's refractive index and with an erbium ion concentration of $6.6 \times 10^{25}$ ions per $\mathrm{m}^{3}$. The power-depended imaginary part of the complex refractive index has been calculated according to [20] and [21] for the unpumped and for the saturated case, where the imaginary part approaches the null value.

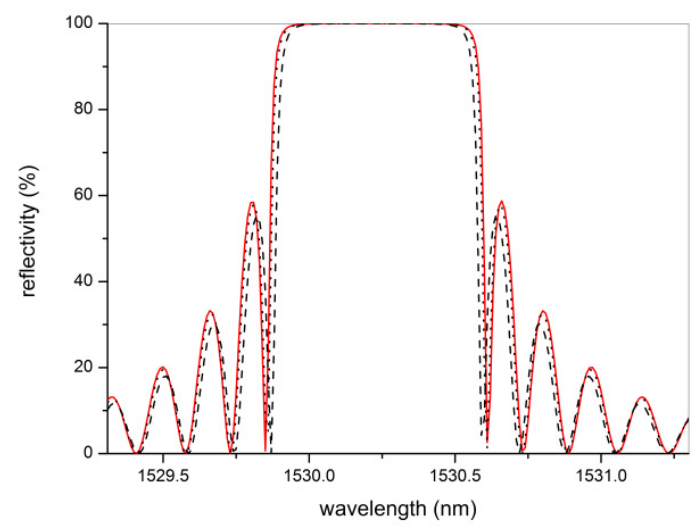

Fig. 3. Convergence of the TLM to CMT method (solid red line) for a typical homogeneous FBG segment. The dashed and dotted line represent the TLM's spectral power reflectivity with $\Delta L=\Lambda / 5$ and $\Delta L=\Lambda / 10$, respectively.

In Fig. 4 the solid lines represent the spectral reflectivity estimated by CMT for the unpumped and pumped cases. It is evident that for the unpumped case, the complex refractive index depicts losses due to negative imaginary part and, as a result, the reflectivity is reduced.

Next, we may approach the same system by applying the TLM. In Fig.4 the dashed lines represent the spectral reflectivity of the ED-FBG with $\Delta L=\Lambda / 10$. The small deviation at the peak reflectivity between the two methods (less than 4\%) may be further decreased if we reduce the layer thickness $\Delta L$ to $\Lambda / 20$ (see dotted lines in Fig.4). Evidently, the application of the TLM requires at least one order higher number of layers than would be used in the CMT method for the simulation of a uniform FBG, and therefore it is more time consuming. Nevertheless, the application of CMT on gratings with non-uniform sinusoidal refractive index requires an increased number of layers and in such cases the CMT computational time is similar to the one experienced with TLM.

Furthermore, the TLM may simulate a non-uniform non- 
sinusoidal grating, where the CMT is not directly applicable. In particular, the inscription of a grating may be subject to deviations from the ideal uniform sinusoidal form on both the inscription depth and the periodicity. Similar deviations may be caused by several factors, such as the accuracy of the phase mask and the uniformity of the UV sensitivity of the fiber's core along the inscription axis.

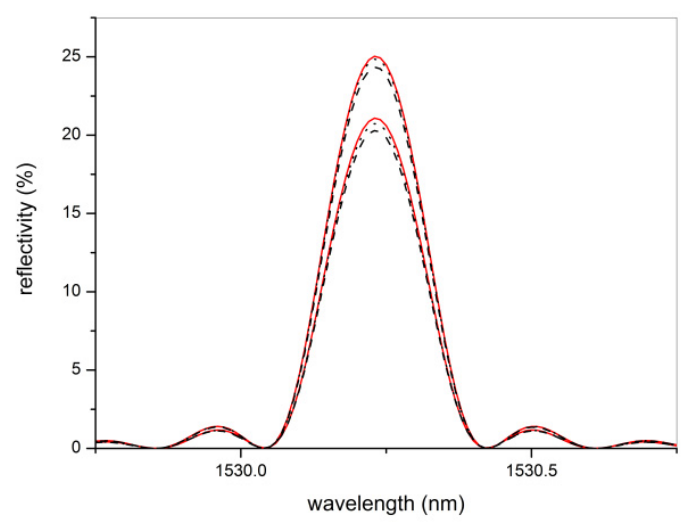

Fig. 4. Convergence of the TLM to CMT method (red solid line) for an Erbium-Doped FBG. The dashed and dotted lines represent the TLM's spectral power reflectivity with $\Delta L=\Lambda / 10$ and $\Lambda / 20$, respectively. The higher (lower) reflectivity represents the pumped (unpumped) FBG.

In order to simulate such deviation scenarios we have introduced a random error to the inscription depth and periodicity by using a numerical random generator that may vary the refractive index accordingly. In Fig.5a, the refractive index variation of a $10 \mu \mathrm{m}$-long section of a grating of length $4 \mathrm{~mm}$ is depicted. The random generator provides a variation of 0 to $-3 \%$ on the inscription depth and of 0 to $+0.0013 \%$ on the inscription periodicity.

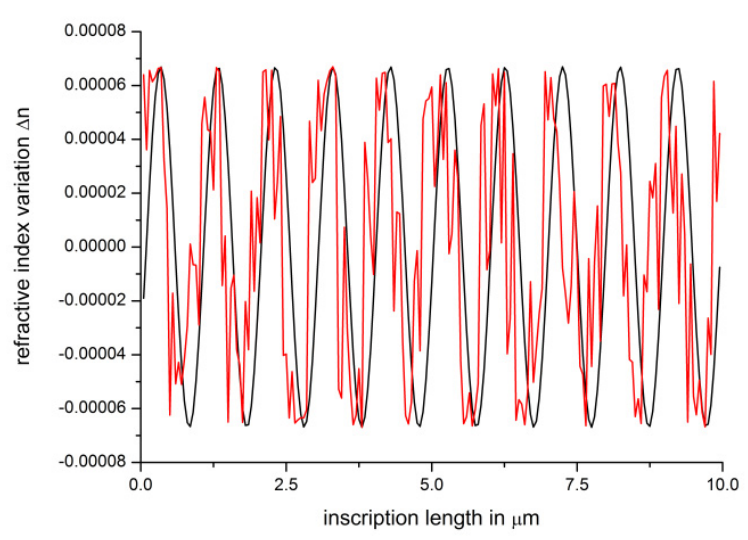

Fig. 5a. The refractive index variation for a length of $10 \mu \mathrm{m}$ from a $4 \mathrm{~mm}$ grating; the ideal sinusoidal inscription (black solid line) and its version with a random variation of 0 to $-3 \%$ on the inscription depth and of 0 to $+0.0013 \%$ on the inscription periodicity (red solid line).

The application of the TLM for a refractive index distribution along the inscription axis, such as the one depicted in Fig.5a, is straight forward without any modification of the model that is described in section 2.B. Simply, by introducing random changes in $\Delta \mathrm{n}$ and $\Lambda$ values as they appear in (8) the deviations from the ideal uniform sinusoidal form are simulated.

The corresponding spectral reflectivities for both the ideal uniform sinusoidal grating and that with the random error are illustrated in Fig.5b. The introduction of the aforementioned random error in the refractive index form may reduce the maximum reflectance by $11.5 \%$ and shift the corresponding wavelength by $0.002 \%$. As a result, the application of the TLM may predict the potential random error encountered during the inscription of the grating.

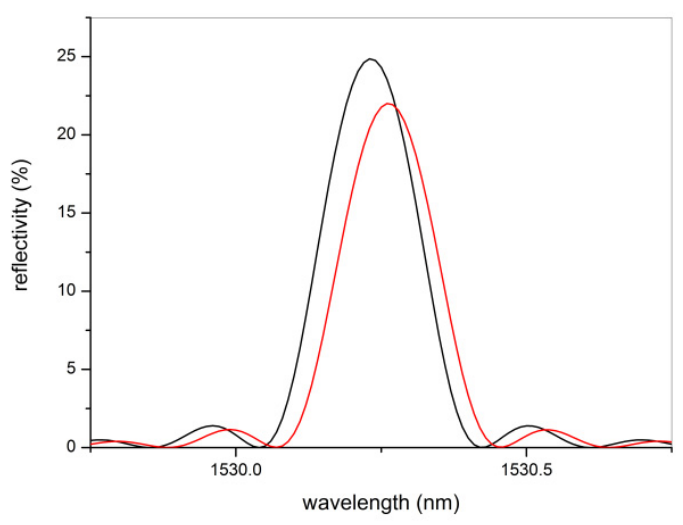

Fig. 5b. The spectral reflectivity of an FBG with ideal inscription (black solid line) versus the spectral reflectivity of the FBG with random variation of the amplitude and periodicity (red solid line).

Although the sensor is the most popular application of the uniform gratings, their use as optical filters has attracted great interest in the field of fiber lasers and optical communications. Particularly, the phase shifted FBGs (PS-FBG) have been utilized as optical filters not only in their typical form of the simple $\pi$-phase FBG, but also in their multiple $\pi$-phase shift version [22]. The analysis of a PS-FBG with the TLM may reveal the straight forward procedure of its application. Considering a PS-FBG, the phase difference $\phi(z)$ of (8) should be activated at the point of the phase shift as depicted in Fig. 6. For a $\pi$-phase shift point in the middle of the grating and for the FBG of Fig.3, the distribution of the series and parallel circuit components are illustrated in Fig. 7.

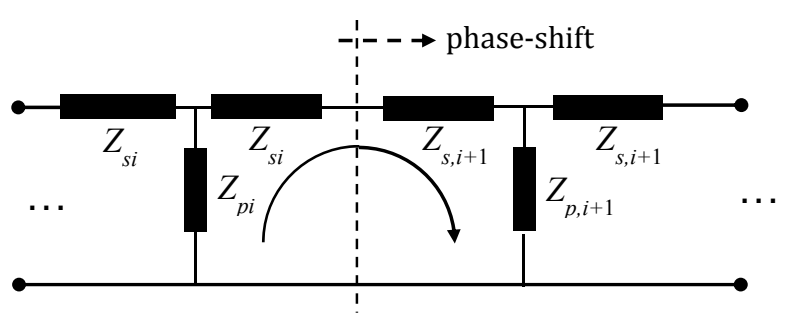

Fig. 6. Equivalent circuit at the phase shift point. 
In Fig.7, it is evident that the sinusoidal variation and the phase shift of the inscribed refractive index directly affect the components of the TLM's equivalent circuit.

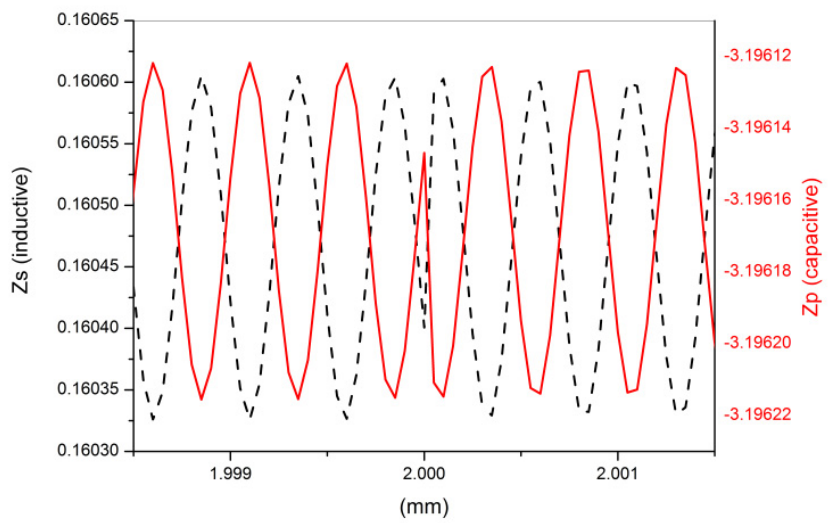

Fig.7. Variation of parallel and series equivalent circuit elements $\mathrm{Zp}$ (solid lines) and Zs (dashed lines) at the phase shift point of a simple $\pi$ phase shift FBG.

The spectral reflection and transmission of the aforementioned simple $\pi$-PS-FBG are illustrated in Fig.8. We have to mention here that the results coincide with the corresponding CMT analysis. Furthermore, should we use $\pi / 2$ phase shift, the transmission wavelength is shifted at a lower value $(1530 \mathrm{~nm})$ as depicted in Fig. 8.

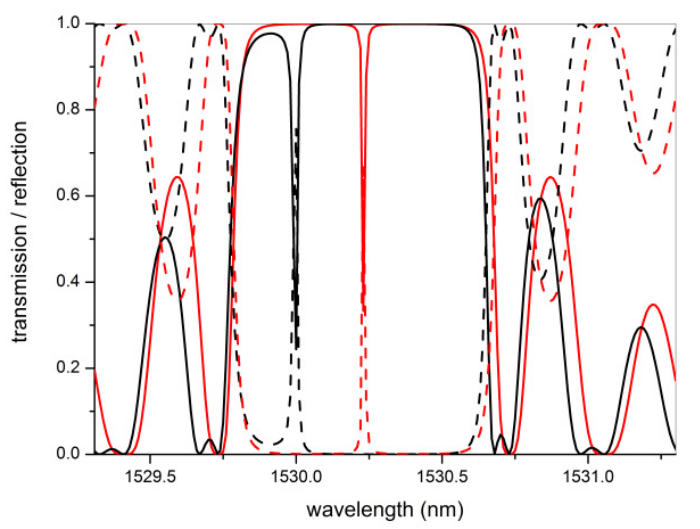

Fig.8. Reflection (solid lines) and transmission (dashed lines) of a simple $\pi$-phase shift FBG (red lines) and a simple $\pi / 2$-phase shift FBG (black lines). TLM and CMT simulations coincide. Both gratings are homogeneous with a length of $4 \mathrm{~mm}$ and inscription depth of $0.04 \%$ while the phase shift point is in the middle of the grating.

Next, we apply the method for the simulation of a double $\pi$-PSFBG. Its spectral reflection is depicted in Fig.9 whereas the grating is symmetric and the distance between the phase shift points is $1.6 \mathrm{~mm}$ (solid line). It is evident that there are two wavelengths that may pass through the grating and the grating's length between the two phase shift points determines the distance between the two wavelengths. Furthermore, experience from electronic bandpass filters may be applied here in order to move the transmitting wavelengths closer to each other. Considering that the grating's length between the two phase-shift points acts as a coupling network, we may increase its length in order to create a transmitting wavelength with a specified bandwidth and reduced ripple (dashed line).

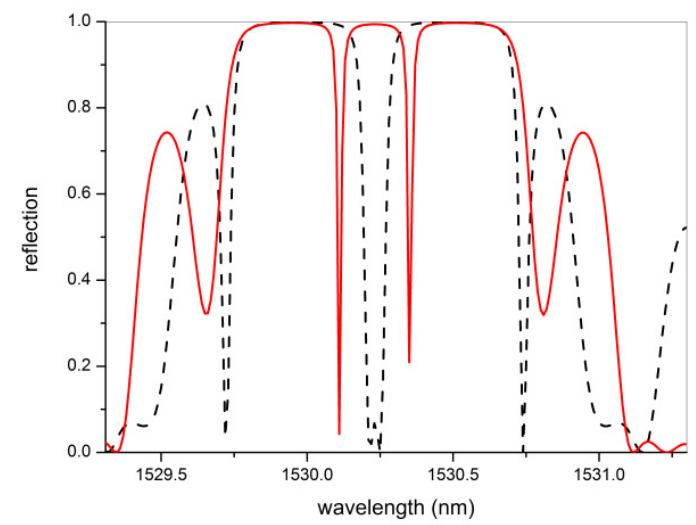

Fig.9. Reflection of symmetric double $\pi$-phase shift FBGs. Both gratings are homogeneous with a length of $4 \mathrm{~mm}$ and inscription depth of $0.04 \%$ whereas the distance between the phase shift points is 1.6 (solid line) and 3.8mm (dashed line) respectively.

Continuing with the narrow band, double $\pi-P S-F B G$ of Fig.9, we analyze its Erbium-doped version. In Fig. 10 the spectral transmission of this grating is depicted, whereas the solid line represents its un-doped version and the dashed line the Erbium-doped version without pumping. From Fig. 10 it is evident that the doped version may increase the loss of the transmission wavelength passband as also presented elsewhere [14]. The switching properties of the ED-PS-FBG may be provided using an external pumping source and increasing the length of the grating.

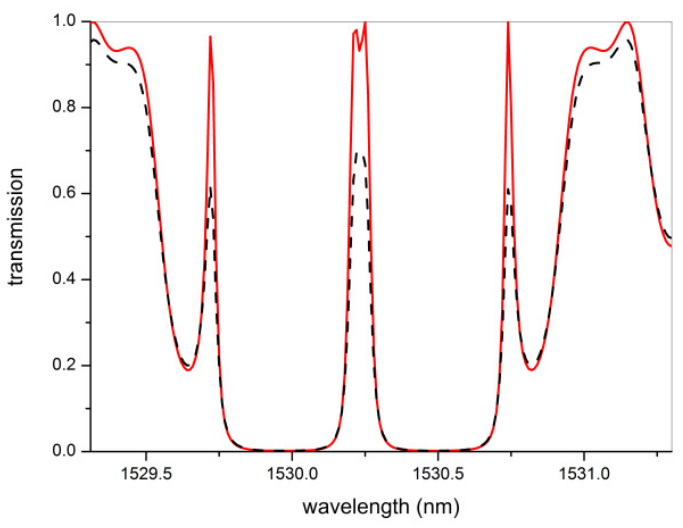

Fig.10. Transmission of a symmetric double $\pi$-phase shift FBG without Erbium doping (solid line) and with doping (dashed line). The gratings are $4 \mathrm{~mm}$ in length with inscription depth of $0.04 \%$ and the distance between the phase shift points is $3.8 \mathrm{~mm}$.

The application of the TLM is also straight forward for the latter ED-PS-FBG configuration, whereas the use of the CMT-TMM requires an extended mathematical analysis [20]. Overall, although the proposed TLM method is more time-consuming, it is easy to implement and to our opinion provides a better physical insight into the problem under simulation. 


\section{Experimental Validation}

In this section an experimental case study for an ED-FBG, which serves as a verification procedure of the proposed method, is presented. The ED-FBG element was fabricated using an Erbium doped single mode fiber (Er110, nLIGHT) with an Erbium-ion concentration of $6.6 \times 10^{25}$ ions per $\mathrm{m}^{3}$. In order to inscribe the grating in the EDF, the core of the EDF was first UVsensitized by the hydrogen loading technique. Finally, the inscription process of the grating with the use of a phase mask resulted in an ED-FBG element, which consists of two parts, one $2 \mathrm{~mm}$ long EDF and one $2 \mathrm{~mm}$ long ED-FBG. The inscription depth is about $0.0043 \%$ of the core's refractive index while the Bragg wavelength is $1530.23 \mathrm{~nm}$.

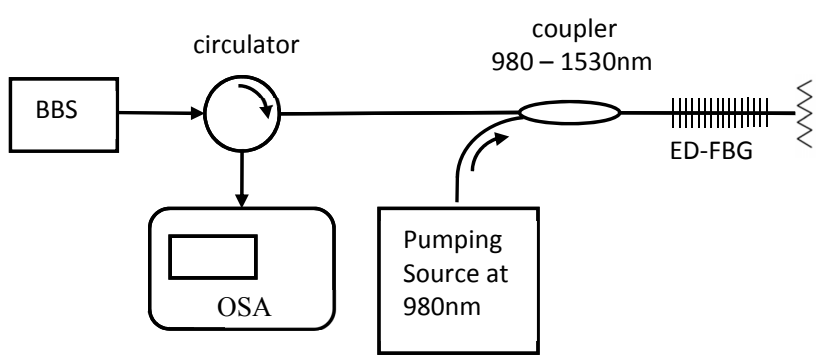

Fig. 11. Experimental setup: BBS is the broad band source and OSA is the optical spectrum analyzer.

For the experimental characterization of this element, the setup of Fig. 11 has been implemented. The broadband light source covers the wavelength spectrum of the ED-FBG reflectivity with almost flat output power, while the power of the pumping source at $980 \mathrm{~nm}$ is adjustable up to $330 \mathrm{~mW}$.

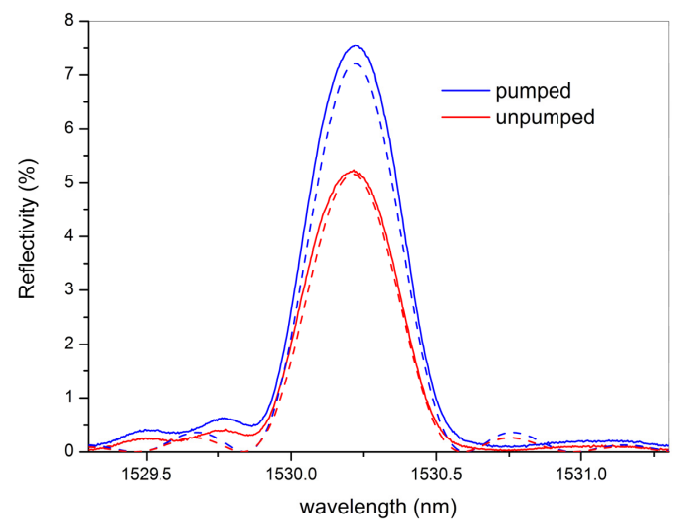

Fig.12. Erbium-Doped FBG spectral power reflectivity for saturating pumping at $980 \mathrm{~nm}$ (blue line) and without pumping (red line) respectively. The dashed lines represent the corresponding TLM results.

In Fig. 12 the reflectivity of the ED-FBG element is depicted for both the unpumped and pumped case (solid lines). In particular, without pumping the erbium inside the core, the refractive index of the core has a negative imaginary part, due to energy absorption. Therefore the first $2 \mathrm{~mm}$ EDF operates as a simple absorber for the incoming signal, while the $2 \mathrm{~mm}$ grating operates as a combined absorber and reflector that reflects back the light but with reduced reflectivity.

Next we applied pumping power from the LD at $980 \mathrm{~nm}$ through the WDM coupler. In order to saturate the erbium inside the element and nullify the imaginary part of the refractive index, the pumping power was properly adjusted.

In this case the reflectivity of the element increases (blue solid line), since there is no absorption by the erbium ions on the signal wavelengths. These two states were simulated by the TLM and the results are also depicted in Fig.12 (dashed lines) for comparison with the experimental data. The agreement between simulation and experimental results are more profound for the unpumped case. This is evident, since for the pumped case the imaginary part of the refractive index may take a small positive imaginary part that represents an insignificant gain. Moreover, the two states reveal the intrinsic characteristic of optical switching of the ED-FBG element.

\section{Conclusions}

An extension of the CMT method based on the transmission line theory is developed for the analysis and synthesis of FBGs. The method provides physical insight, ease and direct implementation in any type of gratings and the ability to simulate random variations of the index inscription. Verification of the method has been conducted both numerically and experimentally. The method could be extended in two mode coupling for long period gratings as part of future work.

\section{Appendix}

By introducing the expansion in terms of ideal modes and considering single mode fiber, we adopt the following equations [15]:

$$
\begin{gathered}
{\left[\frac{d b_{1}}{d z}+j \beta_{1} a_{1}\right\rceil\left(\hat{z} \times H_{1 t}\right)=j \omega \varepsilon_{0}\left(n^{2}-n_{0}{ }^{2}\right) a_{1} E_{1 t}} \\
{\left[\frac{d a_{1}}{d z}+j \beta_{1} b_{1}\right]\left(\hat{z} \times E_{1 t}\right)=\frac{b_{1}}{j \omega \varepsilon_{0}} \frac{n^{2}-n_{0}{ }^{2}}{n^{2} n_{0}{ }^{2}}\left[\nabla_{t} \times\left(\nabla_{t} \times H_{1 t}\right)\right]}
\end{gathered}
$$

The scalar product of (A.1) with $E_{1 t}^{*}$ gives:

$$
\left[\frac{d b_{1}}{d z}+j \beta_{1} a_{1}\right] E_{1 t}^{*}\left(\hat{z} \times H_{1 t}\right)=j \omega \varepsilon_{0}\left(n^{2}-n_{0}^{2}\right) a_{1} E_{1 t}^{*} E_{1 t}
$$

Equivalently, (A.3) may be rewritten as follows:

$$
\left[\frac{d b_{1}}{d z}+j \beta_{1} a_{1}\right] \hat{z}\left(H_{1 t} \times E_{1 t}^{*}\right)=j \omega \varepsilon_{0}\left(n^{2}-n_{0}^{2}\right) a_{1}\left|E_{1 t}\right|^{2}
$$

By expressing $H_{1 t}$ with $E_{1 t}$, (A.4) is simplified as follows: 


$$
\frac{d b_{1}}{d z}=\left(K_{11}-j \beta_{1}\right) a_{1}
$$

where,

$$
K_{11}=-j \frac{k_{0}\left(n^{2}-n_{0}^{2}\right)}{\bar{n}}
$$

In a similar way, the scalar product of (A.2) with $H_{1 t}^{*}$ gives:

$$
\begin{aligned}
& {\left[\frac{d a_{1}}{d z}+j \beta_{1} b_{1}\right] H_{1 t}^{*}\left(\hat{z} \times E_{1 t}\right)=} \\
& =\frac{b_{1}}{j \omega \varepsilon_{0}} \frac{n^{2}-n_{0}{ }^{2}}{n^{2} n_{0}{ }^{2}} H_{1 t}^{*}\left[\nabla_{t} \times\left(\nabla_{t} \times H_{1 t}\right)\right]
\end{aligned}
$$

Deploying the right-hand of (A.7) we have:

$$
\begin{aligned}
& \frac{b_{1}}{j \omega \varepsilon_{0}} \frac{n^{2}-n_{0}{ }^{2}}{n^{2} n_{0}{ }^{2}} H_{1 t}^{*}\left[\nabla_{t} \times\left(\nabla_{t} \times H_{1 t}\right)\right]= \\
& \frac{b_{1}}{j \omega \varepsilon_{0}} \frac{n^{2}-n_{0}{ }^{2}}{n^{2} n_{0}{ }^{2}} H_{1 t}^{*}\left[\nabla_{t} \times\left(j \omega \varepsilon_{0} n_{0}{ }^{2} E_{1 z}\right)\right]= \\
& b_{1} \frac{n^{2}-n_{0}{ }^{2}}{n^{2}} H_{1 t}^{*}\left[\nabla_{t} \times E_{1 z}\right]=b_{1} \frac{n^{2}-n_{0}{ }^{2}}{n^{2}} E_{1 z}\left[\nabla_{t} \times H_{1 t}^{*}\right]= \\
& -j \omega \varepsilon_{0} n_{0}{ }^{2} b_{1} \frac{n^{2}-n_{0}{ }^{2}}{n^{2}} E_{1 z} E_{1 z}{ }^{*}
\end{aligned}
$$

Substituting (A.8) into (A.7) we have:

$$
\begin{gathered}
{\left[\frac{d a_{1}}{d z}+j \beta_{1} b_{1}\right] \hat{z}\left(E_{1 t} \times H_{1 t}^{*}\right)=-j \omega \varepsilon_{0} n_{0}{ }^{2} b_{1} \frac{n^{2}-n_{0}{ }^{2}}{n^{2}}\left|E_{1 z}\right|^{2}} \\
{\left[\frac{d a_{1}}{d z}+j \beta_{1} b_{1}\right] \frac{k_{0}}{\beta_{1}} \sqrt{\frac{\mu_{0}}{\varepsilon_{0}}}\left|H_{1 t}\right|^{2}=} \\
=-j \omega \varepsilon_{0} n_{0}{ }^{2} b_{1} \frac{n^{2}-n_{0}^{2}}{n^{2}}\left|E_{1 z}\right|^{2}
\end{gathered}
$$

or

By integrating (A.10) over the infinite cross section (A.10) is modified as follows:

$$
\frac{d a_{1}}{d z}=\left(k_{11}-j \beta_{1}\right) b_{1}
$$

where,

$$
k_{11}=\frac{\bar{n}\left(n^{2}-n_{0}{ }^{2}\right) n_{0}{ }^{2}}{j k_{0} n^{2}} \frac{\iint \omega^{2} \varepsilon_{0}{ }^{2}\left|E_{1 z}\right|^{2} d A}{\iint\left|H_{1 t}\right|^{2} d A}
$$

The electric and magnetic field intensity across the fiber's cross section in (A.12) as well as the propagation constant $\beta_{1}$ may be calculated either analytically or numerically, by also using a transmission line resonance technique [21].

\section{References}

1. Raman Kashyap, “Fiber Bragg Gratings”, Academic Press (2010)

2. R. Hui, M. O'Sallivan, "Fiber Optic Measurement Technique" Academic Press (2009)

3. G. P. Agrawal, "Applications of Nonlinear Fiber Optics" Academic Press (2001)

4. A. Othonos and K. Kalli, "Fiber Bragg Gratings: Fundamentals and applications in telecommunications and sensing" Artech House (1999)

5. R. Singh, Sunanda, E. K. Sharma, "Gain flattering by long period gratings in erbium-doped fibers," Opt. Comm. 240(1-3), 123-132 (2004)

6. Michiko Harumoto, Masakazu Shigehara, and Hiroshi Suganuma, "Gain-Flattening Filter Using Long-Period Fiber Gratings," J. Lightwave Technol. 20(6), 1027 (2002)

7. S. W. James and R. P. Tatam, "Optical fibre long-period grating sensors: characteristics and application," Meas. Sci. Technol. 14, R49-R61 (2003)

8. J. Hromadka, B. Tokay, S. James, R. P. Tatam, S. Korposh, “Optical fibre long period grating gas sensor modified with metalorganic framework thin films," Sensors and Actuators 221, 891-899 (2015)

9. J. Yang, C. Tao, X. Li, G. Zhu, and W. Chen, "Long-period fiber grating sensor with a styrene-acrylonitrile nano-film incorporating cryptophane A for methane detection," Opt. Express 19(15), 14696 (2011)

10. M. Janczuk-Richter, M. Dominik, E. Rozniecka, M. Koba, P. Mikulic, W. J. Bock, M. Lo's, M. Smietana, J. Niedziółka-Jönsson, "Long-period fiber grating sensor for detection of viruses," Sensors and Actuators 250, 32-38 (2017)

11. Turan Erdogan, "Fiber Grating Spectra” J. Lightwave Technol. 15(8), 1277-1294 (1997)

12. Martin McCall, "On the Application of Coupled Mode Theory for Modeling Fiber Bragg Gratings," J. Lightwave Technol. 18(2), 236 - 242 (2000)

13. Turan Erdogan, "Cladding-mode resonances in short and long period fiber grating filters," J. Opt. Soc. Am. A 14(8), 1760-1773 (1997)

14. Yu Liu, Li Wei, and John W.Y. Lit, "Transmission loss of phase-shifted fiber Bragg gratings in lossy materials: a theoretical and experimental investigation," Applied Optics 46(27), 6770-6773 (2007)

15. D. Marcuse, "Coupled Mode Theory," in Theory of Dielectric Optical Waveguides, 2nd ed., Academic Press (1991).

16. N. Moshonas, G. K. Pagiatakis, P. Papagiannis, S. P. Savaidis and N. A. Stathopoulos, "Application of the transmission line method for the study of highly nonlinear multilayer optical structure," Optical Engineering 53(11), 115106 (2014)

17. R. Feced, M. N. Zervas and M. A. Muriel, "An efficient inverse scattering algorithm for the design of nonuniform fiber Bragg gratings," IEEE J. Quantum Electron. 35(8), 1105-1115 (1999)

18. J. Skaar, L. Wang and T. Erdogan, "On the synthesis of Fiber Bragg Gratings by layer peeling," IEEE J. Quantum Electron. 37(2), 165-173, (2001).

19. X. Chapeleau, D. Leduc, C. Lupi, F. López-Gejo, M. Douay, R. Le Ny, and C. Boisrobert, "Local characterization of fiber-Bragg gratings through combined use of low-coherence interferometry and a layer-peeling algorithm," Applied Optics 45(4), 728-735 (2006).

20. N. A. Stathopoulos, S. P. Savaidis, H. Simos, M. Rangoussi, P. Kervalishvili, "Simulation and properties of Erbium-doped Distributed Bragg Reflectors (ED-DBR) and Fiber Bragg Gratings (ED-FBG)," Optical Fiber Technology 19 (5), 369-377 (2013)

21. S. P. Savaidis, N. A. Stathopoulos, "Propagation characteristics of nonlinear optical fibers with complex refractive index: A transmission line model approach," Opt. Comm. 260, 427-433 (2006).

22. Ye Deng, Ming Li, Ningbo Huang, Ninghua Zhu, "Ka-Band Tunable Flat-Top Microwave Photonic Filter Using a Multi-Phase-Shifted Fiber Bragg Grating," IEEE Photon. Journal 6(4), 5500908 (2014). 
2019-01-04

\section{Transmission line method for the simulation of Fiber Bragg Gratings}

Stathopoulos, N. A.

Optical Society of America

Stathopoulos NA, Savaidis SP, Simos H, et al., (2019) Transmission line method for the simulation of fiber Bragg gratings. Applied Optics, Volume 58, Issue 2, January 2019, pp. 353-360 https://doi.org/10.1364/AO.58.000353

Downloaded from Cranfield Library Services E-Repository 